\title{
PIB, économie de la drogue et territoires
}

L'intégration de l'économie de la drogue dans le calcul du PIB et ses conséquences

GDP, drug economy and territories. Integration of the drug economy in the calculation of GDP and its consequences

\section{Assen Slim}

\section{OpenEdition}

\section{Journals}

Édition électronique

URL : http://journals.openedition.org/echogeo/16085

DOI : $10.4000 /$ echogeo. 16085

ISSN : 1963-1197

Éditeur

Pôle de recherche pour l'organisation et la diffusion de l'information géographique (CNRS UMR 8586)

Référence électronique

Assen Slim, "PIB, économie de la drogue et territoires », EchoGéo [En ligne], Sur le Vif, mis en ligne le 20 novembre 2018, consulté le 30 avril 2019. URL : http://journals.openedition.org/echogeo/16085 ; DOI : 10.4000/echogeo. 16085

Ce document a été généré automatiquement le 30 avril 2019

\section{(2) $\odot \Theta \Theta$}

EchoGéo est mis à disposition selon les termes de la licence Creative Commons Attribution - Pas d'Utilisation Commerciale - Pas de Modification 4.0 International 


\section{PIB, économie de la drogue et territoires}

L'intégration de l'économie de la drogue dans le calcul du PIB et ses conséquences

GDP, drug economy and territories. Integration of the drug economy in the calculation of GDP and its consequences

Assen Slim

"Le PIB mesure tout, sauf ce qui fait que la vie vaut la peine d'être vécue », Robert Kennedy, 18 mars 1968 [cité par Miché, 2010].

\section{Introduction}

1 Comme chaque année, au mois de mai, l'Institut national de la statistique et des études économiques (Insee) révise les comptes nationaux annuels sur les trois dernières années pour tenir compte des informations nouvelles parvenues et non prises en compte. Ainsi, au 30 mai 2018, l'ensemble des données sur la période couverte par les comptes nationaux (1949-2017) a été recalculée en base 2014. A compter de cette date, plusieurs révisions méthodologiques sont devenues effectives, dont les principales portent sur une meilleure prise en compte des flux avec le reste du monde comptabilisés dans la balance des paiements (BdP), la ré-estimation sur quinze ans de la série de consommation finale en services de logement et la révision du compte des administration publique (APU). Bien que significatives, aucune de ces évolutions n'a trouvé d'écho auprès des grands médias. En revanche, l'attention s'est focalisée de manière exclusive sur la dernière révision annoncée par l'Insee, celle portant sur les « séries de production, de consommation finale, d'importation et d'emploi pour tenir compte de la consommation de stupéfiants et des activités liées à cette consommation sur le territoire national, à l'instar des autres pays européens et à la demande d'Eurostat » (Insee, 2018). Pour certains, intégrer l'économie de la drogue dans le calcul du PIB reviendrait à transformer ce dernier en «Produit criminel brut» (Damgé et Laurent, 2014). Or, ces craintes sont infondées, excessives et 
erronées. Elles sont infondées, d'abord, parce que les conséquences de l'économie de la drogue sont déjà comptabilisées dans le PIB. Elles sont excessives, ensuite, parce que la production, les échanges et la consommation de drogue ne représentent qu'une part congrue des richesses produites, échangées et consommées en France sur une année. Elles sont erronées, enfin, parce qu'elles passent à côté des vraies questions posées par le PIB et son mode de calcul actuel.

\section{De quoi le PIB est-il la mesure?}

2 Comme le rappelle l'économiste Denis Clerc (2014), «le produit intérieur brut (PIB) est devenu pour les économistes ce que les déclinaisons sont pour le latiniste ou la puissance du moteur pour l'automobiliste : un point de référence, un élément de base ». Ce constat a le mérite de rappeler à quel point le PIB règne en maitre dans nos sociétés contemporaines. On l'utilise pour mesurer les richesses produites, établir des classements de pays, estimer le niveau de développement des régions du monde, faire des comparaisons spatiales et temporelles, justifier du succès ou de l'échec des politiques économiques. Bref, le PIB est bon à tout dire et, justement, on lui fait tout dire.

3 Ainsi, de manière profondément ancrée dans l'imaginaire collectif, on trouve l'idée reçue selon laquelle plus le PIB est élevé, mieux on se porte. Cela ne manquerait d'ailleurs pas de surprendre ses premiers concepteurs (Simon Kuznets, Jan Tinbergen) pour lesquels le PIB n'a jamais été conçu pour mesurer le bien-être, le bonheur ou une quelconque satisfaction sociale. Pour Jan Tinbergen, par exemple, « le produit national brut n'est pas le bonheur national brut ». Mais s'il n'est pas la mesure du bien-être, alors qu'est-ce que le PIB ?

4 Le PIB est un agrégat de la comptabilité nationale qui peut être calculé de trois façons: côté produits, côté revenus ou côté dépenses. Ce qui a été produit, saisi par la notion de valeur ajoutée (production moins consommations intermédiaires), a bien été distribué sous forme de «revenus» (aux salariés, aux entreprises et à l'Ėtat), et donc utilisé (en dépenses de consommation, d'investissement, dépenses publiques). 
Tableau 1 - Les trois manières de calculer le PIB

\begin{tabular}{|c|c|c|}
\hline côté produits & côté revenus & côté dépenses \\
\hline $\begin{array}{l}\text { somme des valeurs } \\
\text { ajoutées brutes } \\
+ \text { taxe sur la valeur } \\
\text { ajoutée (TVA) } \\
+ \text { droits de douanes } \\
\text { - subventions à } \\
\text { l'importation }\end{array}$ & $\begin{array}{l}\text { rémunérations des } \\
\text { résidents } \\
+\quad \text { excédents rariés } \\
\text { d'exploitation } \\
\text { + impôts liés à la production et } \\
\text { à l'importation }\end{array}$ & $\begin{array}{l}\text { consommation finale } \\
+ \text { formation brute de capital fixe } \\
\text { (FBCF ou investissement) } \\
\text { + variations de stocks } \\
\text { + exportations } \\
\text { - importations }\end{array}$ \\
\hline$=$ PIB & $=\mathrm{PIB}$ & $=\mathrm{PIB}$ \\
\hline
\end{tabular}

Ce qui a été produit a bien été distribué puis dépensé. Côté production, on retranche du PIB les subventions à l'importation qui correspondent à des dépenses réalisées pour importer de la valeur ajoutée étrangère. Côté revenus, le PIB se répartit en trois grands postes : les rémunérations des salariés exerçant leur activité sur le territoire national ; les excédents bruts d'exploitation qui correspondent à la part qui revient à l'entreprise (part elle-même répartie en différents « revenus » : dividendes, loyers, remboursement de prêts, bénéfices) ; les impôts liés à la production et à l'importation qui renvoient à la part qui revient à l'État. Côté dépenses enfin, le PIB est utilisé en dépenses de consommation finale (des ménages, des institutions sans but lucratif, de l'État), en Formation brute de capital fixe (FBCF : investissement public et privé), en constitution d'un stock et en exportations. On retranche habituellement les importations car elles correspondent à de la valeur étrangère importée sur le territoire national. Pour cette dernière colonne du tableau, on parle aussi d'équilibre « ressources/emplois » puisque d'un côté on trouve les ressources (PIB + importations) et de l'autre les emplois (consommation finale, investissement, variation de stock, exportations).

Le PIB ne fait pas la distinction entre les valeurs ajoutées qui favorisent le bien-être et celles qui lui sont néfastes. Il en découle que les accidents de la route, par exemple, par les conséquences qu'ils impliquent (services d'urgence, soins médicaux, rééducation posttraumatique, dépannage et réparation des véhicules), font croître le PIB. Il en va de même des marées noires ou encore des guerres qui, pour certaines d'entre elles, ont été à l'origine de longues périodes de prospérité. Songeons par exemple aux «trente glorieuses » (Fourastié, 1979).

6 Sur ce principe, on peut aisément comprendre que les conséquences de l'économie des stupéfiants (production, distribution, consommation) sont déjà prises en compte dans le calcul PIB sans que cela n'ait jamais choqué personne: coût social de la toxicomanie (structures diverses d'information, de protection, d'aide, de désintoxication), coût des forces de l'ordre chargées de la lutte contre les trafiquants, coût des dispositifs individuels et collectifs de prévention auprès des plus jeunes, coûts des soins hospitaliers ${ }^{1}$ . En plus de ses conséquences, l'Insee intégrera désormais dans le calcul du PIB l'économie de la drogue elle-même.

\section{L'importance du consentement mutuel}

7 La décision d'inclure en mai 2018 l'économie des stupéfiants dans le calcul du PIB intervient quatre ans après la demande d'Eurostat, l'office européen de statistiques. Cette demande avait provoqué d'âpres débats en France, d'autant qu'elle incluait également la prostitution. L'objectif exprimé par Eurostat en 2014 était de faciliter les comparaisons 
entre territoires nationaux de l'Union européenne (UE). En effet, certains pays intègrent déjà la consommation de stupéfiants et/ou la prostitution dans le calcul de leur PIB (Espagne, Royaume-Uni, Italie, Pays-Bas, Allemagne). L'Insee avait alors accepté de donner une estimation des revenus issus des échanges de drogue dans son Revenu National Brut (RNB), qui sert de base de calcul de la contribution française au budget européen, mais pas dans le PIB. La raison invoquée était celle du libre arbitre : « on voit bien que des consommateurs en situation de grande dépendance ne sont plus vraiment en mesure d'exercer leur libre arbitre (et donc, on peut arguer que les transactions ne se font pas vraiment par accord mutuel)» (Damgé, 2018). Toutefois, ce raisonnement pourrait également s'appliquer à d'autres consommations légales et prises en compte dans le PIB (tabac, alcool, jeux de hasard, paris sportifs, jeux vidéo, téléphones portables). Depuis, l'Insee a changé sa position, estimant désormais que « le caractère légal ou illégal, déclaré ou non déclaré, d'une transaction n'est pas un motif recevable pour ne pas la décrire en comptabilité nationale : les comptes nationaux ont en effet vocation à retracer l'ensemble des flux effectifs de revenus » (INSEE , 2018). En d'autres termes, le fait qu'une génération de valeur ajoutée soit légale et déclarée n'est plus un critère déterminant pour sa prise en compte en comptabilité nationale. Ce qui compte dorénavant, c'est l'existence d'un « accord mutuel des parties impliquées » (Ibid.). En ce sens, on peut considérer que le consommateur de stupéfiant a au moins donné son accord lors de son premier achat, c'est-à-dire au moment où il n'était pas encore dépendant. À l'inverse, la prostitution exercée dans la rue par des personnes souvent mineures, généralement en situation irrégulière et sous la coupe de réseaux clandestins s'apparente davantage à une forme d'esclavage sexuel qu'à l'exercice librement consenti d'une activité professionnelle. C'est pour cette raison principale (consentement mutuel) que l'Insee a décidé de ne pas tenir compte de la prostitution, mais d'intégrer, en base 2014, l'économie de la drogue. C'est pour cette même raison que l'Insee incorpore au PIB (depuis la base 2005) la contrebande de tabac mais non le vol de voiture (quand bien même il est générateur de revenus pour les personnes qui s'y adonnent).

\section{Un impact marginal sur le PIB}

Quel sera l'impact de l'intégration de l'économie de la drogue sur le PIB pour la France? Eurostat laisse aux Ėtats membres la possibilité de choisir le mode de calcul qui leur convient. Plutôt que d'opter pour une approche basée sur l'estimation de la consommation (demand-based approach), l'Insee a retenu une approche côté "ressources " (supply-based approach). Comme le soulignent l'Institut national des hautes études sur la sécurité et la justice (INHESJ) et la Mission Interministérielle de Lutte contre les Drogues et les Conduites Addictives (MILDECA), la demand-based approach présente au moins deux grands inconvénients susceptibles de perturber la robustesse des estimations (Ben Lakhdar et al., 2016). Le premier est qu'elle repose sur des enquêtes déclaratives auprès des ménages, or le caractère illicite de la consommation de stupéfiants peut pousser les répondants à sous-estimer leur niveau réel de consommation, voire à ne pas répondre du tout aux questions. Le second a trait aux prix des drogues illicites considérées. L'INHESJ et la MILDECA, qui utilisent un prix médian constaté en France par les forces de l'ordre (OCRTIS) ${ }^{2}$ mais aussi par le réseau TREND de l'observatoire français des drogues et toxicomanies (OFDT), reconnaissent que les chiffres ainsi obtenus masquent non seulement une grande disparité territoriale et organisationnelle mais aussi ce que 
Caulkins et Padman (1993) appellent un «effet discount » lié à l'achat de drogues en larges quantités. C'est pour éviter ces biais que l'Insee a choisi d'appréhender l'économie de la drogue du côté des ressources et non des emplois. Les ressources recouvrent la production résidente des produits (montant de l'autoculture en France), les importations aux prix de base (hors marges commerciales et marges de transport), les marges de commerce (différence entre le prix de revente au consommateur et le prix d'achat des importations à la frontière française par le trafiquant), les marges de transport (coût de transport de la drogue sur le sol français). En comptabilité nationale, les «ressources" correspondent toujours aux «emplois ». En supposant que la drogue n'est ni stockée, ni exportée, ni utilisée comme investissement (hypothèses retenues par l'Insee), alors l'évaluation des ressources disponibles sur le sol français correspond à ce qui est consommé par les ménages résidents. Notons enfin que, dans la nomenclature comptable des produits, l'Insee a choisi d'intégrer les drogues aux produits pharmaceutiques.

On devine toute la difficulté de la supply-based approach. Estimer correctement les différents postes de ressources (production, importation, marges de commerce, marges de transport) implique pour l'Insee d'enquêter au plus près du terrain. De telles évaluations sont difficiles, voire impossibles. L'Insee s'appuie pour le moment sur les études déjà existantes de l'ODFT, de l'INHESJ et de la MIDELCA. Ces dernières estiment les chiffres d'affaires des drogues en France dans une fourchette allant de 1,5 à 3,1 milliards d'euros pour l'année 2010 (tableau ci-dessous) ce qui correspond à une part de $0,07 \%$ à $0,15 \%$ du PIB de cette année. Cet ordre de grandeur situe la France dans la fourchette basse de ce qui est observé dans les autres pays de l'UE. D'après la Commission économique des nations unies pour l'Europe (2018), la part de l'économie de la drogue dans le PIB des pays européens se situerait dans une fourchette allant de 0,13\% (Pologne) à $1,1 \%$ (Royaume-Uni).

Tableau 2 - Récapitulatif des chiffres d'affaires de différents stupéfiant en 2010 en France (en millions d'euros)

\begin{tabular}{|l|l|l|l|}
\hline & Fourchette basse & Moyenne & Fourchette haute \\
\hline Cannabis & 809,7 & 1117,3 & 1424,8 \\
\hline Cocaïne & 503,7 & 902,3 & 1300,8 \\
\hline Héroïne & 204,1 & 266,5 & 328,9 \\
\hline Ecstasy/MDMA & 13,2 & 42,4 & 71,6 \\
\hline Amphétamines & 3,7 & 12,8 & 21,9 \\
\hline Total & $\mathbf{1 5 3 4 , 4}$ & $\mathbf{2} \mathbf{3 4 1 , 3}$ & $\mathbf{3} \mathbf{1 4 8 , 0}$ \\
\hline
\end{tabular}

Source : Ben Lakhdar et al., 2016, p. 3.

De même, les études de l'ODFT, de l'INHESJ et de la MILDECA estiment à un millier de personnes physiques le nombre de «têtes de réseaux » dont l'activité principale est liée à la drogue (cannabis et cocaïne uniquement), ce qui correspond à $0,004 \%$ du total de l'emploi intérieur en France. Il y aurait également 10000 grossistes, 90000 semi- 
grossistes et 130000 revendeurs de rue. En équivalents temps pleins (ETP), on se situerait aux alentours de 21000 ETP, soit 0,08\% du total d'ETP en France en 2014. Les heures travaillées sont estimées à un peu plus de 30 millions d'heures, soit environ $0,07 \% \mathrm{du}$ total d'heures travaillées en France en 2014.

11 En définitive, l'impact sur le PIB de la prise en compte de l'économie de la drogue apparait marginal. La prise en compte elle-même pose des problèmes méthodologiques difficilement surmontables si bien que, pour le moment, l'Insee n'apporte rien de plus que les publications périodiques de l'OFDT. On est loin d'avoir une production de données territorialisés, ventilées par régions ou par métropoles. Ces statistiques territorialisées, si elles devaient voir le jour, ne manqueront pas à leur tour de susciter des débats quant à leur interprétation ${ }^{3}$.

\section{Le PIB, une mesure qui ne dit pas tout}

12 Pour utile qu'ils soient, les efforts pour intégrer l'économie de la drogue dans le calcul du PIB passent à côté des vrais problèmes posés par cet agrégat comptable et des enjeux que représente la recherche d'indicateurs corrigés ou alternatifs.

13 Certains de ces problèmes sont connus de longue date par les statisticiens qui $\mathrm{y}$ ont apporté tant bien que mal des solutions. Il en va ainsi des problèmes posés par l'espace (comparaisons entre PIB de pays différents) et le temps (comparaisons du PIB entre différentes années) auxquels on répond respectivement par les indicateurs en "volume » (à prix constants) et les indicateurs en «PPA » (parité de pouvoir d'achat). Même s'il y a beaucoup à redire sur ces méthodes de correction, elles ont au moins le mérite d'alerter quant aux dangers d'une mauvaise utilisation du PIB. Il en va ainsi également des activités licites qui créée de la «valeur » mais qui sont considérées comme non productives car elles ne sont ni marchandes ni payées : travail domestique privé, bénévolat, troc, loisirs. Arthur C. Pigou remarquait ainsi, au début du XXe siècle, qui si une femme, employée comme domestique par un célibataire, venait à épouser celui-ci, le revenu national en serait diminué car cette femme accomplirait gratuitement les tâches pour lesquelles elle était auparavant rémunérée ${ }^{4}$. En conséquence, le PIB sous-estime considérablement la " richesse " produite dans une société. Toute la difficulté ici consiste à donner un prix à des activités gratuites.

D'autres problèmes sont plus récents et n'ont pour le moment trouvé aucune solution satisfaisante. Il en va ainsi des liens entre PIB et environnement et PIB et inégalités sociales. En effet, le PIB agrège aveuglément les valeurs ajoutées sans tenir compte de leurs potentiels impacts sur les hommes et la nature. Surviennent alors ce que les économistes appellent des externalités sociales (inégalité de distribution des richesses produites) et environnementales (pollutions) négatives. Avec l'aggravation à l'échelle mondiale des inégalités et des dégradations faites aux écosystèmes, la question de leur intégration dans le PIB est devenue cruciale. Comme le précise Patrick Viveret, «plus les questions écologiques et sociétales deviennent prégnantes et plus on se rend compte que nos systèmes d'indicateurs actuels sont contre-productifs » (La Croix, 2009).

15 Les institutions internationales (OCDE, PNUD, Banque mondiale), les Ėtats, les chercheurs, les statisticiens se sont saisis de la question. Pour le moment, deux grandes pistes sont explorées : celle des indicateurs composites (comme le célèbre IDH par exemple) et celle des indicateurs corrigés desquels on a retranché telle ou telle valeur ajoutée jugée 
« néfaste » (indices de «bien-être national net », de « bien-être économique durable », de "progrès réel», de "santé sociale", de "bonheur national brut». Toutefois, ces nouveaux indicateurs butent, à leur tour, sur de nouvelles limites relatives aux choix des critères retenus pour le calcul, aux pondérations, aux imprécisions et manque de fiabilité de données non monétaires et non quantifiables, aux estimations des valeurs des actifs environnementaux. Pour toutes ces raisons, nombreux sont ceux qui estiment que ces initiatives sont sans perspectives de succès et qu'il vaut bien mieux accompagner le PIB d'un tableau d'indicateurs sociaux (taux de mortalité, taux de scolarisation, durée du travail, concentration des revenus, nombre de lits d'hôpitaux), un peu comme ce que fait le PNUD avec son Système satellite des comptes intégrés de l'économie et de l'environnement (SEEA, initié en 1993).

\section{Conclusion}

Se focaliser sur la prise en compte ou non de l'économie des stupéfiants, ou de celle de la prostitution dans le PIB, revient à considérer les problèmes posés par cet indicateur par le petit bout de la lorgnette. Derrière le débat sur nos indicateurs de richesse se cachent des enjeux beaucoup plus fondamentaux quant au modèle de société qui nous souhaitons construire ensemble. Non, la croissance du PIB n'est pas synonyme de développement car elle ne créée pas nécessairement les conditions d'élévation de tout le corps social. Non, la croissance du PIB n'est pas synonyme d'amélioration de notre cadre de vie et du respect des écosystèmes.

Face à ces défis, on peut choisir d'accompagner le PIB d'une batterie d'indicateurs qualitatifs, comme on peut choisir de dépasser le PIB et la logique qui consiste à tout ramener au capital. Car, après tout, toutes les activités qui ont de la "valeur " mais qui n'ont pas de " prix » (comme le bénévolat par exemple) ont toute leur place dans ce qu'on est en droit d'appeler la « richesse » d'une nation. Comme le rappelle Sylvain Coté (2003, p. 41), de tout temps « les indicateurs économiques ont évolué avec les croyances et les circonstances de leur époque » et, ajoutent Jean Gadrey et Florence Jany-Catrice (2012), « il n'y a aucune raison qu'ils n'évoluent pas de nouveau »!

\section{BIBLIOGRAPHIE}

Ben Lakhdar C., Lalam N., Weinberger D., 2016. L'argent de la drogue en France : estimation des marchés des drogues illicites en France. Rapport synthétique de la recherche « Argent de la drogue »à destination de la Mission interministérielle de Lutte contre les Drogues et les Conduites Additives (MILDECA). Paris, INHESJ, $40 \mathrm{p}$.

Bouba-Olga O., Grossetti M., Tudoux B., 2016. On voit des métropoles partout, sauf dans les statistiques. Archives HAL [En ligne]. https://hal.archives-ouvertes.fr/hal-01276897/document

Caulkins J. P., Padman R, 1993. Quantity discounts and quality premia for illicit drugs. Journal of the American Statistical Association, vol. 88, $\mathrm{n}^{\circ}$ 423, p. 748-757. 
Clerc D., 2014. Déchiffrer l'économie. Paris, La Découverte, 462 p.

Coté S., 2003. Rechercher autre chose, mesurer autrement. In Ducroux A.-M. (dir.), Les nouveaux utopistes du développement durable. Paris, Autrement, p. 40-47.

Damgé M., Laurent S., 2014. Sexe, drogue et trafics en tout genre bientôt dans le PIB européen. Le Monde, 6 juin, 2014.

Damgé M., 2018. Comment l'Insee va intégrer le trafic de drogue dans le calcul du PIB. Le Monde, 2 janvier 2018.

Davezies L., Pech T., 2014. La nouvelle question territoriale. Terra Nova, 30 p. [En ligne]. http:// tnova.fr/etudes/la-nouvelle-question-territoriale

Fourastié J., 2011 [ $1^{\text {ere }}$ ed. 1979]. Les trente glorieuses. Paris, Hachette, 288 p.

Gadrey J., Jany-Catrice F., 2012. Les nouveaux indicateurs de richesse. Paris, La Découverte, 128 p.

Insee, 2018a. La prise en compte des stupéfiants dans les comptes nationaux en base $2014.5 \mathrm{p}$.

Insee, 2018b. Communiqué de Presse. 30 mai 2018.

La Croix, 2009. Le PIB un indicateur plus qu'imparfait. La Croix, 8 mars 2009.

Miché J.-C., 2010. L'empire du moindre mal. Essai sur la civilisation libérale. Paris, Flammarion, 205 p.

OFDT, 2018. Synthèse thématique : Cannabis, [En ligne]. https://www.ofdt.fr/produits-et-

addictions/de-z/cannabis/ (consultation du 29/05/2018)

United Nations Development Programme, 1995. Human development report. New York, Oxford

University Press, 230 p. [En ligne] http://hdr.undp.org/sites/default/files/reports/256/

hdr_1995_en_complete_nostats.pdf

United Nations Economic Commission for Europe, 2008. Non-Observed Economy in national Accounts, Survey of Country Practices. New York-Genève, United Nations Publication, 256 p. [En ligne].

https://www.unece.org/fileadmin/DAM/stats/publications/NOE2008.pdf

\section{NOTES}

1. En France, l'Observatoire français des drogues et toxicomanies (OFDT) estime le coût social de la consommation de stupéfiants à 8,7 milliards d'euros par an, contre 120 milliards d'euros environ pour le tabac et l'alcool.

2. Office central pour la répression du trafic illicite de stupéfiants.

3. Voir les débats entre Laurent Davezies et Thierry Pech (2014) qui concentrent leur analyse sur les variations absolues de la dynamique économique des métropoles et Olivier Bouba-Olga, Michel Grossetti et Benoît Tudoux (2016) qui insistent, quant-à-eux sur l'importance du poids des aires urbaines dans les variations mesurées (effet taille).

4. «If a woman employed as a housekeeper by a bachelor were to be married to him, national income would fall, since her previously paid work would now be performed unpaid. But unpaid work goes far beyond housekeeping, and its omission leaves a major gap in National Accounting » (Arthur C. Pigou cité par United Nations Development Programme, 1995, p. 87). 


\section{RÉSUMÉS}

En mai 2018, l'Insee a décidé d'intégrer l'économie de la drogue dans le calcul du PIB. Cette réforme s'inscrit dans un projet plus vaste qui vise à comparer les territoires nationaux au sein de l'UE mais également les territoires régionaux (et/ou métropoles) au sein des pays. Toutefois, elle pose de redoutables problèmes méthodologiques tant pour la récolte des données que pour leur interprétation. De plus, elle n'apporte aucune avancée réelle quant aux véritables interrogations suscitées depuis des années par l'indicateur PIB.

In May 2018, INSEE decided to integrate the drug economy into the calculation of GDP. This reform is part of a larger project aimed at comparing national territories within the EU as well as regional territories (and / or metropolises) within countries. However, it poses serious methodological problems both for the collection of data and their interpretation. Moreover, it does not bring any significant clarification progress on the real questions raised for years by the GDP indicator.

\section{INDEX}

Mots-clés : PIB, économie de la drogue, comptabilité nationale, territoire

Keywords : GDP, drug economy, national account, territory

\section{AUTEUR}

\section{ASSEN SLIM}

Assen Slim, assen.slim@inalco.fr, économiste, est maître de conférences HDR à l'Institut national des langues et civilisations orientales (Langues'O) et professeur associé à l'Essca. Il a récemment publié :

- Slim A., 2016. Comprendre l'économie, un enjeu citoyen : en finir avec les idées reçues. Le Cavalier Bleu, $200 \mathrm{p}$.

- Slim A., 2016. Contrôler mieux pour un monde meilleur, idées reçues sur la tierce partie. Le Cavalier Bleu, $166 \mathrm{p}$.

- Slim A., 2015. Les OGM, c'est le contraire du développement durable. In Andreassian V. et al., Climat, environnement, énergies : 30 idées reçues pour démêler le vrai du faux. Paris, Le Cavalier Bleu, p. 70-76 